Reducti on of $i$ on thermal diffusivity insi de a magnet i c i sl and in JT-60U Tokamak pl asma

\begin{tabular}{|l|l|}
\hline $\begin{array}{l}\text { j our nal or } \\
\text { publ i cat i on t i t l e }\end{array}$ & Physi cal Revi ew Let ter s \\
\hline vol une & Vol . 109 \\
\hline number & I ssue6 \\
\hline page r ange & 065001- 1 - 065001- 4 \\
\hline year & 2012- 08-01 \\
\hline URL & ht t p: //hdl . handl e. net /10655/10140 \\
\hline
\end{tabular}




\title{
Reduction of Ion Thermal Diffusivity Inside a Magnetic Island in JT-60U Tokamak Plasma
}

\author{
K. Ida, ${ }^{1}$ K. Kamiya, ${ }^{2}$ A. Isayama, ${ }^{2}$ Y. Sakamoto, ${ }^{2}$ and JT-60 Team ${ }^{2}$ \\ ${ }^{1}$ National Institute for Fusion Science, Toki, Gifu 509-5292, Japan \\ ${ }^{2}$ Japan Atomic Energy Agency, Naka, Ibaraki-ken 311-0193, Japan
}

(Received 31 May 2012; published 7 August 2012)

\begin{abstract}
A peaked ion temperature profile is observed inside the magnetic island during mode locking after the back transition from $H$ mode to $L$ mode in JT-60U. The thermal diffusivity evaluated inside the magnetic island is $0.1 \mathrm{~m}^{2} / \mathrm{s}$, which is much smaller than that outside the magnetic island by an order of magnitude. The present experiment gives clear evidence that ion heat transport inside a magnetic island can bifurcate and the transport level can be suppressed to the very low level associated with the strong flow shear at the boundary.
\end{abstract}

DOI: 10.1103/PhysRevLett.109.065001

PACS numbers: $52.55 . \mathrm{Fa}, 52.25 . \mathrm{Fi}, 52.50 . \mathrm{Gj}$

Heat transport at a rational surface and/or magnetic island is important in understanding the role of rational surfaces and magnetic islands in the formation of an internal transport barrier [1-3]. This is because there is a strong link between $E \times B$ flow shear to the location of rational surfaces and magnetic islands $[4,5]$. In particle transport, a good confinement in impurity and bulk particle transport inside a magnetic island has been observed as very peaked soft x-ray profiles at the $O$ point of the magnetic island in tokamaks [6-8]; however, the temperature profile is usually only slightly peaked at the $O$ point of the magnetic island [9]. The electron heat diffusivity evaluated with power balance calculations inside the magnetic island shows that electron transport inside the island was comparable to the transport in the ambient plasma [10]. In contrast, perturbation experiments inside the magnetic island using cold pulse or heat propagation show that the transport inside the magnetic island is strongly reduced with respect to the surrounding plasma $[11,12]$. There is a discrepancy in the thermal diffusivity evaluated by pulse propagation and power balance inside the magnetic island in tokamak plasma [12]. It is not clear whether this discrepancy is due to the transient and steady-state heat diffusivity or the difference in the level of temperature gradient (for example, below or above the critical temperature gradient). Although the transport inside the magnetic island is a crucial issue, the characteristics are still open to question.

In general, the temperature gradient inside the magnetic island is small enough to assume a flat temperature profile within the magnetic island. However, this is because the heat flux perpendicular to the magnetic flux surface bypass as the magnetic island and flows through the $X$ point of the magnetic island. Therefore, only the power deposited inside the magnetic island can contribute to the heat flux inside the magnetic island, which is much smaller than the heat flux in the ambient plasma. In order to evaluate the ion heat transport inside the magnetic island, the precise measurement of temperature profiles with high spatial resolution is required. In this Letter, the first observation of a clear peaked ion temperature profile at the $O$ point of a magnetic island and a significant reduction of ion thermal diffusivity inside the magnetic island are described. In JT-60U, the radial profile of ion temperature is measured with the modulation charge exchange spectroscopy [13]. This charge exchange spectroscopy gives ion temperature and toroidal rotation velocity at 310 radial points every $50 \mathrm{~ms}$, which is located separately from the 12 channel electron cyclotron emission (ECE) diagnostic for electron temperature measurements by 80 degrees in toroidal angle and 130 degrees in poloidal angle. Owing to limits in the time resolution of ion temperature measurements, the peaked ion temperature is observed only during the mode locking phase, where the plasma at the magnetic island does not rotate toroidally.

Figure 1 shows the time evolution of the electron temperature measured with the ECE signal near the location of the large magnetic island driven by the neoclassical tearing mode [14]. The plasma rotation stops for $t=6.520-7.207 \mathrm{~s}$ due to mode locking. The heating power of the neutral beam injection (NBI) steps down at $t=6.3 \mathrm{~s}$ from 21 to 15 MW by turning off three perpendicular NBIs, and the back transition from the $H$ mode to the $L$ mode takes place at $t=6.6 \mathrm{~s}$. The radial profiles of the oscillation amplitude of electron temperature have two peaks that correspond to the boundaries of the rotating magnetic island. In this experiment, the oscillation amplitude reaches up to $10 \%$ of the temperature. The location and width of the magnetic island are evaluated from the radial profile of the oscillation amplitude after the disappearance of mode locking at $t=7.27 \mathrm{~s}$ [15]. The mode number of the magnetic island is $m / n=2 / 1$ ( $m$ is poloidal and $n$ toroidal mode number), and the width of the magnetic island evaluated is $0.2(r / a)$ during the oscillation phase. As seen in the expanded plot in Fig. 1(b), the plasma rotation slows down at $t=6.45 \mathrm{~s}$, and finally stops completely at $t=6.520 \mathrm{~s}$ when the $X$ point of the magnetic island is located at the position of the ECE measurements. As seen in the expanded plot in 

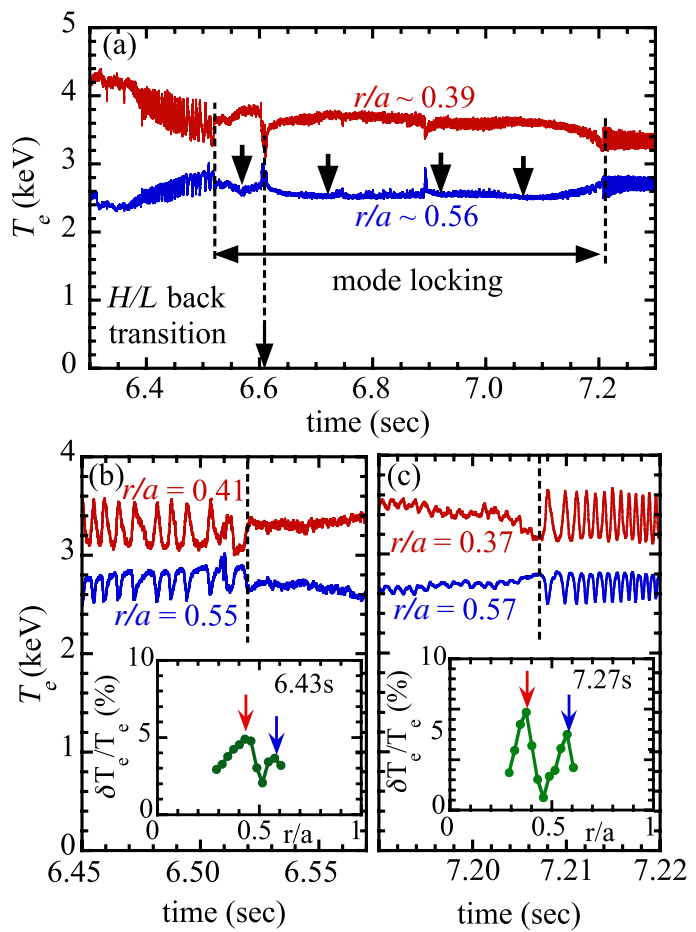

FIG. 1 (color online). Time evolution of (a) electron temperature near the boundary of a magnetic island (at $r / a=0.39$ and $0.56)$ and expanded plot at the (b) onset $(t=6.52 \mathrm{~s})$ and (c) termination ( $t=7.207 \mathrm{~s})$ of the mode locking, when the magnetic island does not rotate (\#49578). The radial profiles of the oscillation amplitude of the electron temperature at $t=6.43$ and $7.27 \mathrm{~s}$ are also indicated.

Fig. 1(c), the plasma starts to rotate gradually from $t=7.20 \mathrm{~s}$ (the $X$ point of the magnetic island is still located at the position of the ECE measurements) to $t=7.207 \mathrm{~s}$ (the $O$ point of the magnetic island is located at the ECE measurements), and then starts to rotate, increasing the rotation speed afterwards. During the mode locking phase, the measurements of electron temperature with ECE are along the $X$ point of the magnetic island, while the measurements of ion temperature profiles with charge exchange spectroscopy are along the $O$ point of the magnetic island.

Figure 2 shows the radial profiles of ion temperature and toroidal rotation velocity in the $H$ mode $(t=6.57 \mathrm{~s})$ and $L$ mode $(t=6.72,6.92$, and $7.07 \mathrm{~s})$ phases. The edge ion temperature at $r / a=0.85$ drops from 3.3 to $2.0 \mathrm{keV}$ at the back transition from the $H$ mode to the $L$ mode phase. After the back transition from the $H$ mode to the $L$ mode phase, the peaked ion temperature profile appears, and this peaked profile is sustained at least $200 \mathrm{~ms}$ without any decay, suggesting significant improvement of ion heat transport inside the magnetic island. The peaking of ion temperature becomes less pronounced and the core rotation increases in the counter direction after one perpendicular NBI and two tangential NBIs in the codirection are turned off at $t=7.0 \mathrm{~s}$ (total NBI power drops from 15 to $9 \mathrm{MW}$ ).

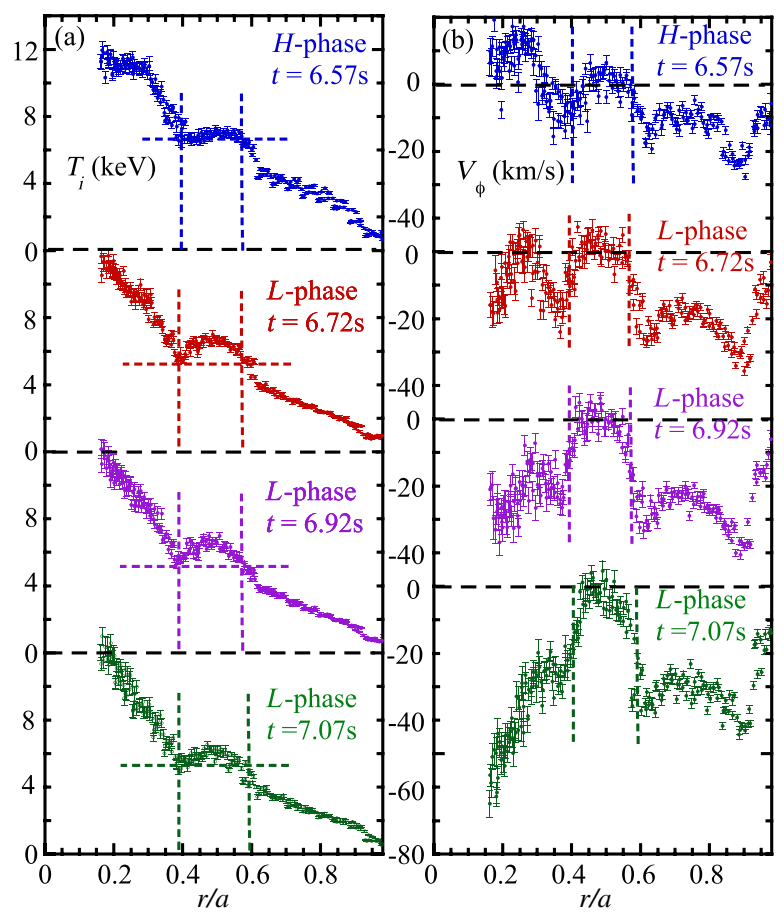

FIG. 2 (color online). Radial profiles of (a) ion temperature and (b) toroidal rotation velocity before $(t=6.57 \mathrm{~s})$ and after ( $t=6.72,6.92$, and $7.07 \mathrm{~s}$ ) the back transition from the $H$ mode to the $L$ mode phase (\#49578).

As seen in the radial profile of toroidal rotation velocity, the plasma inside the magnetic island does not rotate, which is similar to the behavior observed in helical plasmas [5]. Since the plasma outside the magnetic island rotates in the counter direction, there is a strong rotation shear at the boundary of the magnetic island (at $r / a \sim 0.4$ and 0.6). After the tangential beams in codirection are turned off at $t=7.0 \mathrm{~s}$ (switching from balanced NBI to counter NBI), counter rotation increases more and strong rotation shear is observed at the boundary of magnetic island at $r / a \sim 0.6$. This strong rotation shear would contribute to the improvement of confinement at the outer separatrix of the magnetic island $(r / a \sim 0.6)$, where a local large ion temperature gradient is observed. Since there is no toroidal rotation velocity shear inside the magnetic island, one of the candidates for a mechanism for improvement of the ion heat confinement is the convective poloidal flow inside the magnetic island [5]. It is interesting that the magnetic island plays an important role in causing the damping of toroidal rotation and producing rotation shear at the boundary of the magnetic island.

Although the peaked ion temperature profile observed inside the magnetic island is significant $\left[T_{i}(O\right.$ point $)-$ $T_{i}($ separatrix $\left.)=1-2 \mathrm{keV}\right]$, the peaking of the electron temperature is weak $\left[T_{e}(O\right.$ point $)-T_{e}($ separatrix $) \sim$ $0.1 \mathrm{keV}]$. The radial profiles of carbon density are evaluated from the intensity of the charge exchange line of carbon during the $H$ mode and $L$ mode phases as seen in 


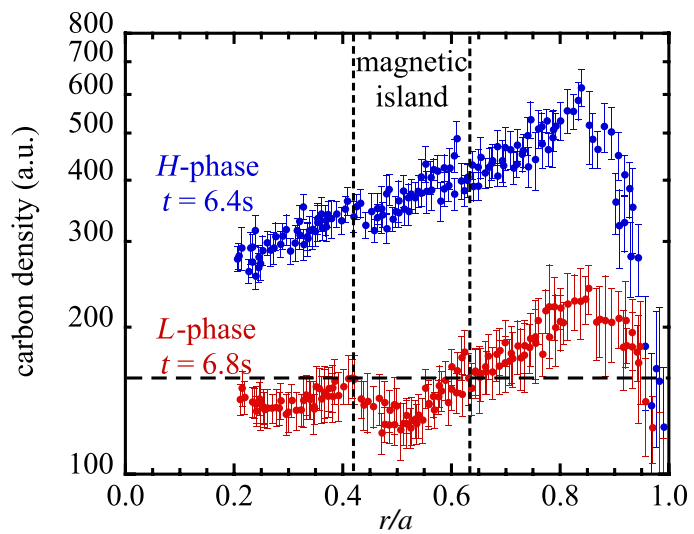

FIG. 3 (color online). Radial profiles of carbon density before $(t=6.4 \mathrm{~s})$ and after $(t=6.8 \mathrm{~s})$ back transition from the $H$ mode to the $L$ mode phase (\#49576).

Fig. 3. After back transition from the $H$ mode to the $L$ mode phase, the carbon density decreases by a factor of 2 . There is no peaking of the carbon density profile in the region of the magnetic island $(r / a=0.42-0.64)$. The carbon density profile is slightly hollow in this discharge, suggesting the outward (from the $O$ point to the separatrix) convection of impurity transport due to the ion temperature gradient $[16,17]$. This is in contrast to the "Snake," where the impurities or bulk particles are confined inside the magnetic island for a long period of the discharge $[6,7]$.

In order to evaluate the thermal diffusivity inside the magnetic island, the flux surface of the magnetic island should be determined. The island flux surface can be expressed with a simple formula of $\Omega=\left(\rho-r_{s}\right)^{2} / w_{h}^{2}-$ $[\cos (m \theta-n \phi)-1] / 2$. Here $r_{s}, w_{h}, \theta$, and $\phi$ are the minor radius of the $O$ point of the magnetic island, the half width of the magnetic island, the poloidal angle, and the toroidal angle, respectively. The island flux surface label is $\Omega=1$ at the separatrix and $\Omega=0$ at the $O$ point. In this analysis, the plasma is divided into three areas: (i) the inner region outside the magnetic island $(r / a<0.42)$, (ii) the region inside the magnetic island ( $0.42<r / a<0.63)$, and (iii) the outer region outside the magnetic island $(0.62<r / a)$; the heat flux for each region is calculated by integrating the heat deposition in the divided region with the boundary condition of continuity of integrated heat flux as $\int Q_{i}^{(i i i)}(r / a=$ $0.63) d S=\int Q_{i}^{(\mathrm{i})}(r / a=0.42) d S+\int Q_{i}^{(\mathrm{ii})}(\Omega=1) d S$. Because of this condition, there are jumps of heat flux normalized by density, and hence the thermal diffusivity at the separatrix of $\Omega=1$. Because the area of the magnetic island is much smaller than that outside the magnetic island, the heat flux inside the magnetic island is smaller than that outside the magnetic island by an order of magnitude. However, as seen in Fig. 4(a), the temperature gradient inside the magnetic island is comparable or even larger than that outside the magnetic island.

The thermal diffusivity near the magnetic axis is low $\left(0.1 \mathrm{~m}^{2} / \mathrm{s}\right)$ and around $1 \mathrm{~m}^{2} / \mathrm{s}$ at half of the plasma minor

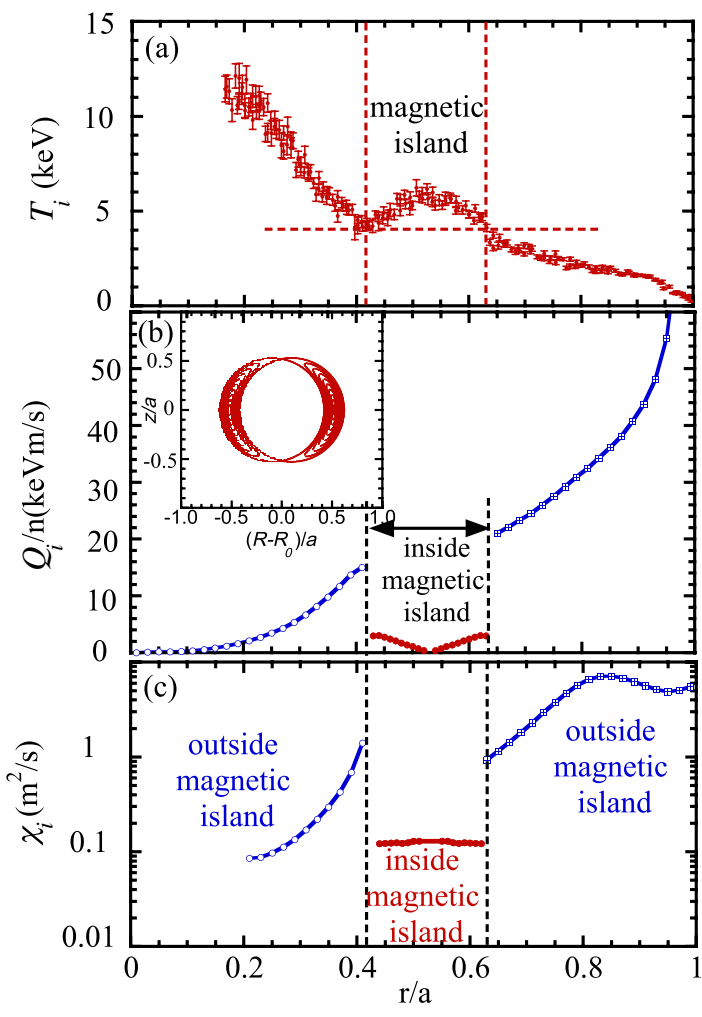

FIG. 4 (color online). Radial profile of (a) ion temperature, (b) heat flux normalized by density, and (c) ion thermal diffusivity in the $L$ mode phase $(t=6.77 \mathrm{~s})$. The magnetic flux surface model used to evaluate the heat flux is also indicated (\#49576).

radius and increases sharply up to $5 \mathrm{~m}^{2} / \mathrm{s}$ toward the plasma periphery. The thermal diffusivity inside the magnetic island is constant in space and very low $\left(\sim 0.1 \mathrm{~m}^{2} / \mathrm{s}\right)$, while that outside the magnetic island is much higher $\left(\sim 1 \mathrm{~m}^{2} / \mathrm{s}\right)$. This result is different from the experimental results in TEXTOR, where the transport inside the island is comparable to the transport in the ambient plasma [10]. The reduction of ion thermal diffusivity inside the magnetic island is triggered by back transition from the $H$ mode phase to the $L$ mode phase. Since the heating power deposited in region (i) flows to region (iii) through the $X$ point of the magnetic island, the global confinement time drops when the large magnetic island appears. However, the drop of confinement time is not due to the poor confinement inside the magnetic island surrounded by the separatrix.

After the back transition, the ion temperature at the separatrix $(\Omega=1)$ drops, causing a transient peaking of the ion temperature. If there is no reduction of ion transport, this peak should disappear within a few tens of ms. Because the reduction of thermal diffusivity simultaneously takes place, the peaked ion temperature can be sustained by a small heat flux inside the magnetic island. Figure 5 shows the time evolution of the ion temperature gradients at both sides of the peaked ion temperature 


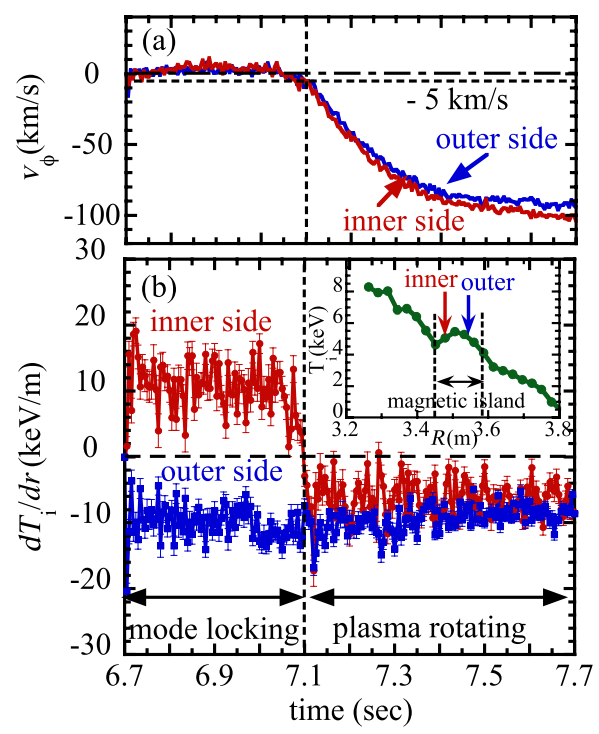

FIG. 5 (color online). Time evolution of (a) toroidal rotation velocity and (b) ion temperature gradient inner and outer sides of the peaked profile inside the magnetic island. The location of the inner and outer sides of peaked profile are indicated by arrows in the radial profile of the ion temperature at $t=6.73 \mathrm{~s}$ (\#49715).

profile. In this measurement, the charge exchange spectroscopy is operated in the fixed mode, where the time resolution is improved up to $5 \mathrm{~ms}$ but with 31 spatial channels. The positive gradient indicates the peaked ion temperature inside the magnetic island. The ion temperature gradient at the inner side of the peaked profile is $\sim 10 \mathrm{keV} / \mathrm{m}$ and almost constant in time until the plasma starts to rotate at $t=7.1 \mathrm{~s}$. After the plasma toroidal rotation velocity exceeds $5 \mathrm{~km} / \mathrm{s}$, which corresponds to the rotation frequency of $200 \mathrm{~Hz}$, the peaked structure cannot be observed even though it exists because of the smoothing in time (the time resolution of the ion temperature measurement is not enough when the mode locking disappears).

An interesting issue is why the transport can bifurcate inside the magnetic island. When the temperature profile is flat inside the magnetic island, the turbulence is small because there is no, or only a small, temperature gradient. However, there is also no flow shear because of having no temperature gradient. In contrast, when the ion temperature becomes peaked, the turbulence is driven by the temperature gradient, but a convective poloidal flow [5] driven by the temperature gradient can contribute to the suppression of turbulence. Therefore, similar to the bifurcation between the $L$ mode and the $H$ mode, the magnetic island can have two possible states, one, the state with a flat temperature profile without flow shear, and the other, the state with peaked temperature with flow shear.
In a reverse field pinch (RFP) plasma, a peaked temperature profile inside the magnetic island can be observed [18]. Local power balance calculations suggest a reduced thermal transport within this island from $10^{3} \mathrm{~m}^{2} / \mathrm{s}$ to $3 \times 10 \mathrm{~m}^{2} / \mathrm{s}$. This reduction of thermal diffusivity is due to change in topology from the stochastic state to helical states. In contrast, there is no change in topology in the magnetic flux surfaces, and the thermal diffusivity drops from $1 \mathrm{~m}^{2} / \mathrm{s}$ to $0.1 \mathrm{~m}^{2} / \mathrm{s}$ due to change in turbulence states in the JT-60U experiment. The present experiment gives clear evidence that there are two states of transport inside the magnetic island. Transport analysis inside the magnetic island shows a state in which thermal diffusivity inside the magnetic island is significantly reduced to a level much lower than the transport in ambient plasma by an order of magnitude.

We would like to thank the technical staff for their efforts in supporting the JT-60U experiment. This work is partly supported by a Grant-in-Aid for Specially Promoted Research (21224014) of MEXT Japan.

[1] Y. Koide et al., Phys. Rev. Lett. 72, 3662 (1994).

[2] E. Joffrin et al., Nucl. Fusion 43, 1167 (2003).

[3] T. Estrada et al., Nucl. Fusion 47, 305 (2007).

[4] C. Hidalgo et al., Plasma Phys. Controlled Fusion 42, A153 (2000).

[5] K. Ida et al., Phys. Rev. Lett. 88, 015002 (2001).

[6] K. Ida, R. J. Fonck, R. A. Hulse, and B. Leblanc, Plasma Phys. Controlled Fusion 28, 879 (1986).

[7] A. Weller, A. D. Cheetham, A. W. Edwards, R. D. Gill, A. Gondhalekar, R. S. Granetz, J. Snipes, and J. A. Wesson, Phys. Rev. Lett. 59, 2303 (1987).

[8] P. C. de Vries, A. J.H. Donne, S. H. Heijnen, C. A. J. Hugenholtz, A. Kramer-Flecken, F. C. Schuller, and G. Waidmann, Nucl. Fusion 37, 1641 (1997).

[9] P. C. de Vries, G. Waidmann, A. Krämer-Flecken, A. J. H. Donné, and F. C. Schüller, Plasma Phys. Controlled Fusion 39, 439 (1997).

[10] I. G. J. Classen et al., Phys. Rev. Lett. 98, 035001 (2007).

[11] S. Inagaki et al., Phys. Rev. Lett. 92, 055002 (2004).

[12] G. W. Spakman et al., Nucl. Fusion 48, 115005 (2008).

[13] K. Ida, Y. Sakamoto, M. Yoshinuma, S. Inagaki, T. Kobuchi, G. Matsunaga, and Y. Koide, Rev. Sci. Instrum. 79, 053506 (2008).

[14] A. Isayama et al., Nucl. Fusion 49, 055006 (2009).

[15] A. Isayama, Y. Kamada, T. Ozeki, and N. Isei, Plasma Phys. Controlled Fusion 41, 35 (1999).

[16] M. R. Wade, W. A. Houlberg, and L. R. Baylor, Phys. Rev. Lett. 84, 282 (2000).

[17] K. Ida et al., Phys. Plasmas 16, 056111 (2009).

[18] H. D. Stephens, Phys. Plasmas 17, 056115 (2010). 